\title{
Colaboração entre governos e organizações da sociedade civil em resposta a situações de emergência
}

\author{
Mário Aquino Alves 1 \\ Marcelo Marchesini da Costa ${ }^{2}$ \\ 1 Fundação Getulio Vargas / Escola de Administração de Empresas de São Paulo, São Paulo / SP - Brasil \\ 2 Insper Instituto de Ensino e Pesquisa, São Paulo / SP - Brasil
}

\begin{abstract}
A pandemia da COVID-19 é um exemplo de grande emergência que desafia a administração pública ocasionalmente. A despeito da variedade de eventos dessa natureza, recai sobre o governo, majoritariamente, a responsabilidade pelas ações emergenciais nesses momentos. Organizações da Sociedade Civil (OSCs) podem, no entanto, contribuir para uma resposta rápida e adequada a tais ocorrências. Neste artigo, são discutidas as características das OSCs que as habilitam a contribuir para as medidas de emergência adotadas pelos governos. Analisa-se também a possibilidade de ampliação dos arranjos colaborativos entre governos e OSCs.

Palavras-chave: COVID-19; organizações da sociedade civil; emergência; colaboração.

\section{Colaboración entre gobiernos y organizaciones de la sociedad civil en respuesta a situaciones de emergencia}

La pandemia de COVID-19 es un ejemplo de gran emergencia que ocasionalmente desafía a la administración pública. A pesar de la variedad de eventos de esa naturaleza, el gobierno es el principal responsable de las acciones de emergencia en esos momentos. Sin embargo, las organizaciones de la sociedad civil (OSC) pueden contribuir a una respuesta rápida y adecuada a estas emergencias. Este artículo analiza las características de las OSC que las califican para contribuir a las medidas de emergencia adoptadas por los gobiernos. También se analiza la posibilidad de ampliación de los acuerdos de colaboración entre gobiernos y OSC.

Palabras clave: COVID-19; organizaciones de la sociedad civil; emergencia; colaboración.

\section{The collaboration between governments and civil society organizations in response to emergency situations}

The COVID-19 pandemic is an example of a large-scale emergency that defies public administration. There is a variety of large-scale emergency events, and the government is responsible for responding to such situations. Civil Society Organizations (CSOs) can contribute to a fast and appropriate response to these emergencies. This article discusses the characteristics of CSOs that qualify them to contribute to the government's emergency responses. We also analyze possible collaborative arrangements between governments and CSOs.

Keywords: COVID-19; civil society organizations; emergency; collaboration. 


\section{INTRODUÇÃO}

Catástrofes e grandes emergências, como fenômenos climáticos extremos, ataques terroristas e epidemias, exigem ações rápidas de modo a preservar vidas, limitar danos e possibilitar uma rápida superação da crise. Usualmente, cobra-se do governo, em seus diversos níveis, essa resposta. É natural e correto que seja assim, pois o governo é o principal responsável pela salvaguarda do interesse público. No entanto, não seria viável o governo manter sempre à disposição uma estrutura que só se faz necessária em momentos de grandes catástrofes. A crise global gerada pela COVID-19 é um claro exemplo disso: o enfrentamento da pandemia requer inúmeros leitos e equipamentos hospitalares que seriam supérfluos durante um período de normalidade.

Uma possível alternativa para responder de imediato a desafios deste gênero é aproveitar a infraestrutura, os recursos humanos e os processos instituídos no setor privado. Uma crise como a da COVID-19, no entanto, traz também enormes desafios à realização dos objetivos econômicos e à sustentabilidade das empresas. Há, ainda, um grupo importante de organizações da sociedade civil, as (OSCs), cuja função social destaca-se nos momentos de grande comoção pública. Há diversos exemplos de mobilização espontânea da sociedade civil para minimizar os danos da COVID-19 e que poderiam ser ampliados com apoio e coordenação governamental.

O objetivo deste trabalho é indicar como governos de todos os níveis podem se beneficiar das OSCs para aprimorar suas respostas a diversos tipos de emergências. Neste artigo discutimos como a resposta a uma grande emergência exige dos governos um papel central, porém com grandes oportunidades para ganhos de escala e capilaridade na resposta, por meio de parcerias e colaborações com a sociedade civil.

Inicialmente, apresenta-se o contexto atual das OSCs no Brasil; depois, discutem-se brevemente as teorias sobre relações intersetoriais. Na seção seguinte, analisa-se o contexto de grandes emergências. Por fim, apresentam-se recomendações para ações e políticas nesse contexto e as conclusões do artigo.

\section{CONTEXTO DE ORGANIZAÇÕES DA SOCIEDADE CIVIL NO BRASIL}

Apresentamos o contexto das OSCs no Brasil com base em algumas dimensões centrais: a financeira, a institucional, a gerencial e a política. Da perspectiva financeira, tem havido uma grande redução de recursos de cooperação internacional destinados às OSCs brasileiras desde o início dos anos 2000 (Marchesini da Costa, 2019). Além disso, o avanço de governos em vários níveis com discursos hostis às OSCs, eleitos em 2018, compromete a continuidade de programas de financiamento governamental dessas organizações. Para as OSCs, o processo de restrições do financiamento, acompanhado de desconfiança sobre a seriedade de suas atividades, estende-se por quase dez anos, desde o início do século XXI (Mendonça, Alves, \& Nogueira, 2016).

Na dimensão institucional, o desenho da regulação das OSCs no Brasil desempenha um papel central. Aqui, entende-se por regulação das OSCs a criação de legislação primária (constituição e leis) ou secundária (portarias, instruções normativas, decisões administrativas) por parte do Estado, somada às contribuições e aos mecanismos autorregulatórios do setor (Breen, Dunn, \& Sidel, 2019). Apesar de ter havido um avanço do ponto de vista da regulação, com a aprovação do Marco Regulatório das Organizações da Sociedade Civil (MROSC) em meados da década passada (Appe \& Marchesini da Costa, 2016), o ecossistema de controle governamental (que compreende tanto os tribunais de contas quanto corregedorias e mesmo o Ministério Público [MP]) não 
separa a malversação de fundos públicos dos problemas de dificuldades em prestação de contas, levando à criminalização de todas as OSCs (Souza \& Oliveira, 2020). Além disso, a regulação das parcerias entre OSCs e Estado no Brasil é muito centrada nas linguagens e práticas concebidas do ponto de vista do Estado, o que implica pouca confiança mútua entre os agentes (Peci, Oquendo, \& Mendonça, 2020).

O aspecto gerencial evidencia certas fragilidades das OSCs. Tanto no Brasil, quanto internacionalmente, via de regra, as OSCs não possuem as competências gerenciais específicas para captação de recursos e seu desenvolvimento institucional (Armani, 2008; Bish \& Becker, 2016). Grande parte delas minimiza a relevância da boa gestão, ainda que um grupo influente de organizações do terceiro setor, em geral ligadas a fundações e grupos empresariais, destaque-se na adoção de ferramentas de excelência gerencial (Marchesini da Costa, 2019). No entanto, a filantropia corporativa - que compreende o investimento social privado no Brasil - concentra recursos em projetos próprios, alinhados às estratégias corporativas e muitas vezes alheios a outras pautas das OSCs brasileiras (Mendonça, Alves, \& Nogueira, 2013; Pagotto, Belinky, Macedo, \& Yamahaki, 2016). De toda forma, é necessário disseminar boas práticas de gestão no universo de OSCs brasileiras.

Por fim, é importante salientar que o momento político do país, no qual predomina um pensamento conservador e hostil às OSCs, sobretudo àquelas que lidam com direitos humanos, cria mais dificuldade para que o cidadão médio aproxime-se dessas organizações, quer seja para o exercício do voluntariado, quer seja para doar recursos (Abdal et al., 2019; Alves, Nogueira, Pineda, \& Campos, 2019). Esse momento político deve afetar de forma diversa as OSCs brasileiras, até mesmo pela própria diversidade do setor. Por exemplo, organizações religiosas, que constituem 35\% das OSCs do Brasil, tendem a ser afetadas de forma diferente entre si - se a organização for ligada a uma linha progressista ou conservadora - e em relação aos 12\% de OSCs formados por associações profissionais (Instituto Brasileiro de Geografia e Estatística [IBGE], 2016).

A despeito das dificuldades e limitações apresentadas, ao longo das últimas décadas houve um crescimento do número de OSCs, chegando a aproximadamente 780.000 organizações em abril de 2020 (Instituto Econômico de Pesquisa Aplicada [IPEA], 2020). As OSCs estão presentes em milhares de municípios; muitas atuam e beneficiam uma região mais ampla, formando, também, redes de atuação com outras organizações (Marchesini da Costa, 2017). Vale novamente lembrar que grande parte das OSCs brasileiras são organizações religiosas, possuem, portanto, importante papel na comunidade (Marchesini da Costa, 2017). Por fim, os serviços prestados e a missão dessas organizações são diversos, porém, grande parte está voltada a atividades sociais, como desenvolvimento social, assistência e saúde (Ipea, 2020; Marchesini da Costa, 2017), indicando um potencial de contribuição justamente em questões relevantes no enfrentamento da COVID-19.

Antes de aprofundar a discussão sobre a participação de OSCs na resposta a grandes emergências, a próxima seção apresenta elementos relevantes sobre a origem de OSCs e de como elas se relacionam com o Estado.

\section{RELAÇÕES INTERSETORIAIS}

Comumente, o termo intersetorial refere-se à iniciativa de interesse a diferentes políticas setoriais, como políticas para a juventude, por exemplo (Marcondes, Sandim, \& Diniz, 2018), contudo, neste artigo, utiliza-se o conceito no sentido de relações entre diferentes setores da economia. Mais 
especificamente, as relações entre o Estado e o terceiro setor ${ }^{1}$. Alguns autores (Weisbrod, 1978) entendem que as organizações da sociedade civil emergem de "falhas no mercado" (market failure) para satisfazer a demanda por bens públicos, ou seja, por bens que deveriam ser democraticamente disponibilizados, gratuitamente ou não. No modelo clássico, essa "falha de mercado" cria condições para que o governo intervenha e ofereça estes bens públicos (Lecy \& Van Slyke, 2012; Smith \& Lipsky, 1993; Young, 2000). Nas democracias, em que há uma grande diversidade de opiniões sobre quais bens públicos devem ser produzidos e ofertados, e somente a opinião do eleitor médio é considerada, existiriam nichos de demanda que não seriam satisfeitos por uma "falha de governo" (government failure) (Smith \& Lipsky, 1993; Weisbrod, 1978). Nestas condições, a sociedade civil mobiliza-se para produzir e ofertar serviços públicos que não podem ser assegurados nem pelo mercado, nem pelo governo (Steinberg, 2003; Weisbrod, 1978).

Se as teorias de falha de mercado e falha de governo enfatizam a demanda não satisfeita por bens públicos, outras teorias escrutinam a "falha de oferta" (supply failure) (James, 1990; Steinberg, 2003). Tomando como necessária, mas não suficiente, a ideia de que existe uma demanda não atendida por bens públicos, a teoria da "falha de oferta" enfatiza que alguns agentes individuais, ou coletivos, seriam incentivados a criar organizações sem fins lucrativos para atender tal demanda, o que faria emergir um conjunto de "empreendedores sociais" (James, 1990). Por exemplo, onde mais de uma religião exerce influência, existem condições para uma "competição religiosa" por novos seguidores (Salamon \& Anheier, 1998). Nessa competição por novos fiéis, congregações, por exemplo, criam bens e serviços, tais como provisão de educação, assistência social e assistência médica (James, 1990; Salamon \& Anheier, 1998).

Os modelos teóricos mostrados anteriormente partem de uma premissa básica: a existência de organizações da sociedade civil depende, entre outras coisas, da ausência (ou ineficiência) de um Estado e de um mercado que atendam às necessidades das pessoas em termos econômicos. Ou seja, mercados e governos efetivos no atendimento às demandas da sociedade poderiam prescindir da existência de um setor privado sem fins lucrativos. Seguindo esta lógica, há teorias que compreendem a existência da Sociedade Civil (ou do terceiro setor) como uma forma residual da existência do Estado, ou como um elemento complementar na regulação da ordem capitalista (Abzug \& Webb, 1999). A literatura em geral sobre Welfare State, por exemplo, dá pouca atenção à questão da Sociedade Civil ou do Terceiro Setor (Salamon \& Anheier, 1998). Quando muito, a Sociedade Civil é tratada como um conjunto de instituições pré-modernas de bem-estar social que, da mesma forma que as famílias, as organizações religiosas e outras formas organizacionais de caridade, perdeu sua função assistencial primordial com o desenvolvimento do capitalismo (Flora \& Heidenheimer, 1981). Desse ponto de vista, as instituições sociais mais arcaicas não conseguiriam atender a provisão social requerida, provocando a emergência de um tipo de Estado que se encarregaria desse tipo de provisão (Offe, 1984). Por esse motivo, algumas sociedades não teriam desenvolvido mais do que um Estado de Bem-Estar "residual", uma vez que mantiveram muito da sua provisão social sob formas institucionais arcaicas (Titmuss, 1974).

Se as teorias mais clássicas sobre o Estado de Bem-Estar tendem a tratar a Sociedade Civil de maneira residual, ou como instituições arcaicas que perderam o sentido com o capitalismo, mais recentemente este panorama tem mudado. Possivelmente em virtude da crise do Estado de Bem-Estar

\footnotetext{
${ }^{1}$ Aqui assumimos como Alves (2004), que o “terceiro setor" é parte constitutiva da sociedade civil, mais voltada para a prestação de serviços.
} 
Social, que parece não atender mais as necessidades relativas a bens públicos, muitos pesquisadores passaram a considerar a Sociedade Civil como um conjunto de organizações e instituições que se colocam par a par com o mercado e com o Estado na produção de bens e serviços de fruição pública. Segundo Lester Salamon (1987), tanto as visões economicistas quanto as reguladoras partem de um paradigma de conflito entre Estado e Sociedade Civil (ver Najam, 2000). Em outras palavras, os diversos autores somente conseguem perceber espaços de conflito e competição entre os setores estatal e sem fins lucrativos. No entanto, Salamon (1987) defende que, simultaneamente às esferas conflituosas, também emergem tendências de parceria e interdependência entre o Estado e a Sociedade Civil. Aliás, a interdependência seria mais comum do que o próprio conflito.

Normalmente as OSCs são mais ágeis do que o governo no campo social, e seu potencial de mobilização política funciona como um estímulo à ação do Estado e, simultaneamente, inclui-as no processo de provisão de bens e serviços (Boris, 2006; Frumkin, 2002). Entretanto, as OSCs têm limitações inerentes no atendimento de demandas por bens públicos - voluntary failure ou "falha do setor não lucrativo" - , principalmente no que diz respeito à geração de recursos que sustentam suas atividades e, em muitos casos, devido a formas paternalistas de gestão, em que há dificuldades para empreender políticas sociais mais abrangentes (Salamon, 1995). Por esse motivo, segundo Salamon (1995), criamse possibilidades de cooperação entre Sociedade Civil e Estado para resolver determinados problemas sociais. Essas possíveis relações dependeriam de diversos fatores, em especial de natureza ideológica: por pressões de natureza política, o Estado por vezes deixa de intervir diretamente no suprimento de determinado bem público, outorgando a provisão deste às OSCs. Na visão de determinados setores, tais organizações teriam maior legitimidade política para essa prestação de serviços.

Algumas teorias da cooperação entre Estado e Sociedade Civil (Najam, 2000; Salamon, 1987; Young, 2000) consideram que, diferentemente dos ensinamentos econômicos, ou mesmo na visão mais tradicional sobre Estado de Bem-Estar, quanto maior for o campo de atuação do Estado, maior será a possibilidade do desenvolvimento da Sociedade Civil (Abzug \& Webb, 1999; Salamon \& Anheier, 1998), com o Estado funcionando como o maior financiador de suas atividades. De fato, as parcerias entre OSCs e governos no Brasil são amplas e com resultados variados, segundo o tipo de organização e a região do país onde atuam (Marchesini da Costa, 2017). Exemplos mais específicos apontam, entre outros resultados relevantes, que OSCs mais bem estruturadas são capazes de sugerir propostas de ação a governos locais (Ribeiro, Andion, \& Burigo, 2015), e que OSCs cumprem importante papel no aprimoramento da democracia ao monitorar a ação de governos (Moreira, Teodósio, \& Ayres, 2020). Especificamente na área de saúde, organizações sociais prestam grande parte dos serviços nos maiores municípios do país (Ditterich, Moysés, \& Moysés, 2012).

Tendo sido apesentadas as organizações da sociedade civil e do terceiro setor, é necessário agora compreender o papel de OSCs em resposta a grandes emergências.

\section{ESPECIFICIDADES DE GRANDES EMERGÊNCIAS E ARRANJOS DE GOVERNANÇA COLABORATIVA}

Emergências como acidentes automobilísticos e pequenos incêndios ocorrem todos os dias. Há organizações públicas voltadas prioritariamente para lidar com essas situações, como o Corpo de Bombeiros e a Defesa Civil2 . Grande emergências, no entanto, diferenciam-se pela escala e por criar

\footnotetext{
${ }^{2}$ Em vários países, Corpo de Bombeiros e Defesa Civil são organizações da sociedade civil com forte suporte do Estado.
} 
um contexto específico ao qual as organizações e os processos usuais não conseguem se adequar (Henderson, 2014). Diversos autores destacam a importância da colaboração entre diferentes setores para responder a grandes emergências de vários tipos, desde ataques terroristas (Comfort \& Kapucu, 2006) a desastres naturais (Simo \& Bies, 2007). Estudos nos Estados Unidos indicam que a Cruz Vermelha e o Exército da Salvação são as principais fontes de assistência a vítimas de desastres daquele país (Waugh \& Streib, 2006). Gestores públicos em momentos de emergência devem estar preparados para aproveitar os recursos financeiros, administrativos e políticos que a rede de parceiros não governamentais pode oferecer em resposta à situação (Waugh \& Sylves, 2002).

O primeiro desafio imposto pelas grandes emergências é mobilizar rapidamente essa rede de potenciais parceiros da sociedade civil para auxiliar nas ações. Inúmeros exemplos indicam que, com ou sem a coordenação do governo, o terceiro setor tende a se mobilizar para responder às grandes emergências. Pouco tempo após o início da pandemia da COVID-19, o Grupo de Institutos, Fundações e Empresas (GIFE), que representa a filantropia institucionalizada no Brasil, desenvolveu a plataforma "Emergência COVID-19" de apoio a projetos da sociedade civil no enfrentamento da pandemia (https://emergenciacovid19.gife.org.br/); a Associação Brasileira de Organizações Não Governamentais (Abong), que representa algumas das OSCs de defesa de direitos, lançou a "Rede Solidária” (https://www.redesolidaria.org.br/), que congrega ações de engajamento cívico, doação e advocacy. Simultaneamente, novos empreendimentos também surgiram como resposta rápida e emergencial à pandemia, como o projeto Favelas Contra o Coronavírus (https://www.facebook.com/ favelascontraocoronavirus), ou as mobilizações comunitárias em Paraisópolis, na cidade de São Paulo. O Quadro 1 lista apenas uma pequena parte destas iniciativas, de modo a evidenciar sua amplitude e variedade de focos.

\section{QUADRO 1 EXEMPLO DE INICIATIVAS DE OSCS NO BRASIL EM RESPOSTA À PANDEMIA DA COVID-19}

\begin{tabular}{|c|c|}
\hline OSC & Breve Descrição da Iniciativa \\
\hline Central Única de Favelas (CUFA) & $\begin{array}{l}\text { Complementação de renda de } \mathrm{R} \$ 120 \text { para mulheres com filhos em comunidades } \\
\text { durante a pandemia. }\end{array}$ \\
\hline Médicos sem Fronteiras (MSF) & Atendimento médico às populações mais vulneráveis. \\
\hline Ação da Cidadania & Doação de alimentos. \\
\hline $\begin{array}{l}\text { Serviço Franciscano de } \\
\text { Solidariedade (Sefras) }\end{array}$ & Doação de equipamentos de proteção e higiene pessoal. \\
\hline $\begin{array}{l}\text { Articulação de Povos Indígenas do } \\
\text { Brasil (APIB) }\end{array}$ & Doação de alimentos, remédios e material de higiene para aldeias indígenas. \\
\hline Plataforma Enfrente (várias OSCs) & $\begin{array}{l}\text { Iniciativa de Matchfunding (fundo complementa com } \mathrm{R} \$ 2 \text { para cada } \mathrm{R} \$ 1 \text { doado) com } \\
\text { centenas de projetos participantes. }\end{array}$ \\
\hline Comunitas & Doação de equipamentos como respiradores para hospitais da rede pública. \\
\hline
\end{tabular}

Fonte: Elaborado pelos autores com base em pesquisa ao website das organizações. 
O Quadro 1 destaca iniciativas com baixa ou nenhuma colaboração do Estado. Assim, cabe apontar como podem ser efetivadas ações colaborativas em situações de emergência.

Um misto de mecanismos formais, como termos de parcerias e contratos, e mecanismos informais, como relações pessoais e acordos informais, pode ser utilizado para acomodar todos os potenciais parceiros (Waugh \& Sylves, 2002). Estudos sobre governança colaborativa analisam os aspectos que agilizam os arranjos intersetoriais. Destaca-se, por exemplo, a necessidade de um claro objetivo comum, um marco regulatório adequado, boas experiências em relações preexistentes e lideranças comprometidas (Bryson, Crosby, \& Stone, 2015; Shaw \& Goda, 2004).

A rápida mobilização de esforços, entretanto, pode não ser o maior dos desafios. Nem toda colaboração tem resultados positivos (O’Donovan, 2019). Um abrigo para moradores de rua gerido por uma OSC que acelere a propagação da COVID-19, por exemplo, seria mais parte do problema do que da solução. São necessários, portanto, estrutura, processos e, sobretudo, no momento inicial, comunicações efetivas entre os parceiros (Bryson et al., 2015), e esclarecimentos sobre ações que possam auxiliar na resposta a uma situação de emergência. $\mathrm{O}$ investimento público é fundamental para viabilizar uma estratégia de comunicação que reforce e esclareça esse objetivo comum (Comfort \& Kapucu, 2006).

Por fim, salienta-se que, em situações de desastres ou crises, ações imediatas são importantes, por parte do Estado, para garantir a sustentabilidade institucional e financeira da própria Sociedade Civil (Shaw \& Goda, 2004). Isso é necessário não só para a garantia, no longo prazo, da capacidade de atendimento capilar das OSCs (Shaw \& Goda, 2004), mas para a manutenção da confiança mútua nas comunidades (Tilly, 2008) e do próprio regime democrático (Lin, 2015).

\section{RECOMENDAÇÕES DE AÇÕES E POLÍTICAS}

Com base nos argumentos apresentados, destacamos a seguir algumas proposições de ações e políticas públicas a serem implementadas por governos em diferentes níveis. Ressaltamos ainda que as medidas a seguir são aspectos gerais de uma estratégia de resposta a grandes emergências, podendo auxiliar na resposta à pandemia da COVID-19 e a outros eventos deste porte. Há, evidentemente, particularidades relacionadas a modalidades diferentes de emergência, como uma pandemia, uma grande enchente ou um grande incêndio, mas essas particularidades não são consideradas. A proposição refere-se a cuidados básicos, portanto, genéricos, a serem considerados pelos governos em qualquer situação, para um ótimo aproveitamento da capacidade das OSCs.

\section{1) Liderança governamental comprometida com boas relações intersetoriais}

Modelos de governança colaborativa enfatizam a importância de boas relações preexistentes entre os diferentes atores para que a colaboração seja bem sucedida (Bryson et al., 2015). Ações e declarações que demonstrem desconfiança e animosidade de líderes governamentais com relação a OSCs geram resistência por parte destas em colaborar com o governo. É preciso que o governo mantenha um diálogo constante, reconhecendo a importância das OSCs nas comunidades em que atuam. Há excelentes propostas da sociedade civil para o enfrentamento da pandemia da COVID-19, como a de 'Quartos da Quarentena', da OSC Minha Sampa (https://www.quartosdaquarentena.minhasampa.org.br/), que 
prevê a utilização de hotéis como abrigo temporário da população de rua ou de outros segmentos vulneráveis durante a pandemia. É fundamental, para isso, que o exemplo e o direcionamento dos líderes governamentais sejam inequívocos no reconhecimento da importância das OSCs. De nada adianta um corpo técnico e burocrático comprometido se não houver respaldo no discurso e nas ações dos líderes políticos do governo.

\section{2) Flexibilização de processos e controles}

A diversidade das OSCs implica em boa parte das organizações com baixa capacidade organizacional ou mesmo com propensão a desvios (Ebrahim, 2003; Smillie, 1995). Governos experientes em parcerias com OSCs possuem informações que lhes permitem diferenciar e excluir esses casos, sobretudo quando existem espaços de interlocução já estabelecidos e habituais, como conselhos (Mendonça, Medeiros, \& Araújo, 2019). Os momentos de grandes emergências, no entanto, não são propícios aos procedimentos usuais de contratação e controle de parceiros. Em estudo recente, Souza e Oliveira (2020) mostram que responder aos procedimentos de controle usuais, como o Siconv (agora Plataforma+Brasil) ainda é um desafio para muitas OSCs. É preciso rapidez para acionar colaborações combinada com segurança para que os parceiros ajam sem o receio de serem responsabilizados por detalhes das regras de controle (Mendonça et al., 2019). Para isso é preciso que fique absolutamente claro quais são as prioridades durante a crise e na retomada das atividades (Blanco, 2015).

\section{3) Transparência e governo aberto}

Transparência e abertura por parte do governo são condições prévias em situações de grande emergência. Entretanto, é preciso cuidado para que as ações tomadas em resposta à situação se enquadrem e reforcem os mecanismos de governo já existentes. Por exemplo, flexibilizar o controle burocrático para as medidas de emergências não significa não disponibilizar prontamente as informações sobre compras e contratos em portais de compras públicas. Assim se possibilita accountability, permitindo a identificação de desvios e falhas que poderiam comprometer a legitimidade das respostas a grandes emergências.

\section{4) Comunicação efetiva}

Possivelmente, o primeiro e mais importante ato do governo para viabilizar ações da sociedade civil que auxiliem na resposta a grandes emergências é estabelecer canais de comunicação que sejam: rápidos, constantemente atualizados, claros, facilmente acessíveis e com mensagens convergentes. A dificuldade em acessar informações oficiais ou a falta de uma comunicação governamental genuinamente dialógica, que permita a identificação de informações corretas, pode gerar sentimentos de raiva e descrença na sociedade e mobilizá-la para o confronto (Kang, Kim, \& Cha, 2018), além de desencorajar a ação colaborativa de OSCs ou desencadear ações que atrapalhem e dificultem as respostas. 


\section{5) Reforço na atuação dos conselhos de políticas públicas}

Arranjos de governança vão além da colaboração na implementação de políticas públicas. O constante relacionamento entre agentes governamentais e representantes de OSCs em conselhos de políticas públicas pode contribuir para gerar confiança e vínculos positivos entre esses atores. Para isso, no entanto, é preciso que os conselhos funcionem efetivamente, com encontros regulares, condução democrática das reuniões e na escolha dos membros, debates respeitosos sobre as questões relevantes. Há uma diversidade muito grande de conselhos, em virtude de suas políticas setoriais e de seus contextos (Buvinich, 2014), e, ainda, conselhos municipais tendem a ser mais democráticos que os nacionais (Gomes, 2015). Ainda assim, a garantia de uma comunicação dialógica efetiva com a sociedade passa pela ativação dos conselhos, sobretudo em momentos de crise (Kang et al., 2018), contribuindo para deliberações de natureza mais democrática (Gomes, 2015).

\section{6) Editais focalizados da filantropia institucional (fundações e institutos empresariais e familiares) para ampliar capacidade das OSCs}

No que concerne às ações filantrópicas organizadas no Brasil em razão da pandemia da COVID-19, como doações emergenciais ${ }^{3}$, notam-se esforços focalizados em mitigar os efeitos da pandemia em áreas vulneráveis e em reforçar a capacidade de atuação das OSCs das próprias comunidades. Há, portanto, por parte da filantropia institucional, o desafio de lançar editais específicos para a recuperação da capacidade institucional das OSCs, de modo a promover sua sustentabilidade e desenvolvimento comunitário.

\section{CONCLUSÃO}

Este artigo analisa como as OSCs podem mobilizar recursos humanos e organizacionais relevantes em resposta aos casos de grandes emergências. A capilaridade, o conhecimento específico e a orientação das missões sociais e humanitárias podem facilitar e potencializar as ações em resposta aos problemas causados pela pandemia da COVID-19. Estudos sobre arranjos colaborativos intersetoriais apontam que esse potencial, utilizado mundialmente em tempos de normalidade, pode também ser mobilizado em caso de grandes emergências.

As situações de emergência podem impulsionar novas relações entre governos e OSCs, gerando oportunidades de contato e vínculos de confiança entre essas partes. No entanto, em grande medida, a qualidade da resposta depende de ações e políticas anteriores a esses eventos. O bom proveito da capacidade das OSCs para apoiar ações emergenciais depende de condições de relacionamento entre governo, OSCs e sociedade. Essas ações perdem força se não forem implementadas em conjunto. A Figura 1 representa a interação entre as ações e políticas sugeridas neste trabalho.

\footnotetext{
${ }^{3}$ Até o momento que terminávamos a presente versão do artigo (16 de maio de 2020), o Monitor das Doações da COVID 19 da ABCR (https://www.monitordasdoacoes.org.br/) contabilizava um total de R \$4.873.927.567,00, cuja maior parte é proveniente da filantropia institucional para ações de combate à epidemia. Quase todas as doações foram feitas em recursos financeiros ou em espécie para governos ou organizações de atenção à saúde (privadas ou públicas).
} 
RAP | Colaboração entre governos e organizações da sociedade civil em resposta a situações de emergência

FIGURA 1 POLÍTICAS E AÇÕES EM COLABORAÇÃO COM OSCS COMO RESPOSTAS ÀS EMERGÊNCIAS

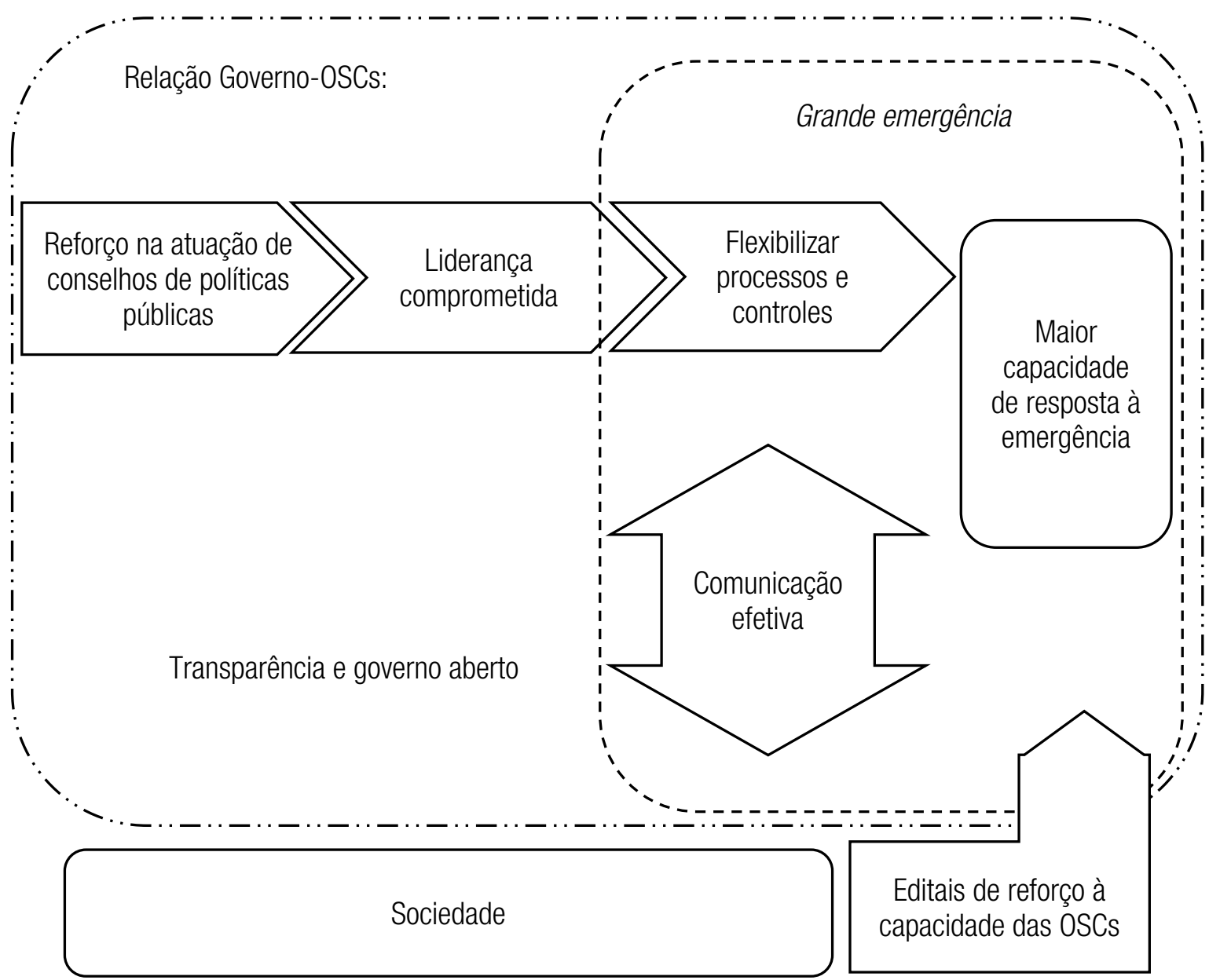

Fonte: Elaborada pelos autores.

Um arranjo colaborativo entre governos e OSCs não só ampliaria a rede de prestação de serviços e respostas em caso de grandes emergências, como poderia aprimorar as políticas e as ações adotadas. 


\section{REFERÊNCIAS}

Abdal, A., Alves, M. A., Nogueira, F. A., Pineda, A., Campos, P. H., Calixto, G., \& Campos, G. (2019). Pesquisa Comportamental sobre Doadores de Alta Renda. São Paulo, SP: Conectas e CEAPG.

Abzug, R., \& Webb, N. J. (1999). Relationships between nonprofit and for-profit organizations: a stakeholder perspective. Nonprofit and Voluntary Sector Quarterly, 28(4), 416-431.

Alves, M. A. (2004). O conceito de sociedade civil: em busca de uma repolitização. Organização e Sociedade, 11, 141-154.

Alves, M. A., Nogueira, F. A., Pineda, A., \& Campos, P. H. (2019, maio). Doadores de Alta Renda e Organizações de Direitos Humanos no Brasil. In Anais do $8^{\circ}$ Encontro de Administração Pública da ANPAD, Fortaleza, CE. Recuperado de http://www.anpad. org.br/eventos.php?cod_evento $=6 \&$ cod_edicao_ subsecao $=1650 \&$ cod_evento_edicao $=94 \& \operatorname{cod}$ _ edicao_trabalho $=26147$

Appe, S., \& Marchesini da Costa, M. (2016). Waves of Nonprofit Regulation and Self-Regulation in Latin America: Evidence and Trends from Brazil and Ecuador. In O. B. Breen, A. Dunn, \& M. Sidel (Eds.), Regulatory Waves Comparative Perspectives on State Regulation and Self-Regulation Policies in the Nonprofit Sector (pp. 154-175). Cambridge, UK: Cambridge University Press.

Armani, D. (2008). Mobilizar para transformar: a mobilização de recursos nas organizações da sociedade civil. São Paulo, SP: Editora Peirópolis.

Bish, A., \& Becker, K. (2016). Exploring expectations of nonprofit management capabilities. Nonprofit and Voluntary Sector Quarterly, 45(3), 437-457.

Blanco, D. V. (2015). Disaster governance in the Philippines: Issues, lessons learned, and future directions in the post-Yolanda super typhoon aftermath. International Journal of Public Administration, 38(10), 743-756.

Boris, E. T. (2006). Nonprofit Organizations in a Democracy: Varied Roles and Responsibilities. In E. T. Boris, \& C. E. Steuerle (Eds.), Nonprofits \& Government: Collaboration and Conflict (2nd ed., pp. 1-36). Washington, DC: Urban Institute.

Breen, O. B., Dunn, A., \& Sidel, M. (2019). Riding the regulatory wave: reflections on recent explorations of the statutory and nonstatutory nonprofit regulatory cycles in 16 jurisdictions. Nonprofit and Voluntary Sector Quarterly, 48(4), 691-715.

Bryson, J. M., Crosby, B. C., \& Stone, M. M. (2015). Designing and implementing cross-sector collaborations: needed and challenging. Public Administration Review, 75(5), 647-663.

Buvinich, D. P. R. (2014). O mapeamento da institucionalização dos conselhos gestores de políticas públicas nos municípios brasileiros. Revista de Administração Pública, 48(1), 55-82.

Comfort, L. K., \& Kapucu, N. (2006). Interorganizational coordination in extreme events: The World Trade Center attacks, September 11, 2001. Natural Hazards, 39(2), 309-327.

Ditterich, R. G., Moysés, S. T., \& Moysés, S. J. (2012). O uso de contratos de gestão e incentivos profissionais no setor público de saúde. Cadernos de Saúde Pública, 28(4), 615-625.

Ebrahim, A. (2003). Accountability in practice: Mechanisms for NGOs. World Development, 31(5), 813-829.

Flora, P., \& Heidenheimer, A. J. (1981). The development of welfare states in Europe and America. Piscataway, Nova Jersey, USA: Transaction Publishers.

Frumkin, P. (2002). On being nonprofit: A conceptual and policy primer. Massachussets, USA: Harvard University Press.

Gomes, E. G. M. (2015). Conselhos gestores de políticas públicas: aspectos teóricos sobre o potencial de controle social democrático e eficiente. Cadernos EBAPE.BR, 13(4), 894-909.

Henderson, A. (2014). The Critical Role of StreetLevel Bureaucrats in Disaster and Crisis. In R. Schwester. (Ed.), Handbook of Critical Incident Analysis (pp. 217-235). Nova York, NY: M. E. Sharpe.

Instituto Brasileiro de Geografia e Estatística. (2016). As Fundações Privadas e Associações sem Fins Lucrativos no Brasil - 2016. Rio de Janeiro, RJ: Autor. Recuperado de https://sidra.ibge.gov.br/ tabela/6914\#resultado

Instituto de Pesquisa Econômica Aplicada. (2020). Mapa de Organizações da Sociedade Civil. Rio de Janeiro, RJ: Autor. Recuperado de https://mapaosc. ipea.gov.br/resultado-consulta.html 
James, E. (1990). Economic theories of the nonprofit sector: A comparative perspective. In H. K. Anheier \& W. Seibel, The Third Sector, Comparative Studies of Non-Profit Organizations (Series De Gruyter Studies in Organization, 21, pp. 21-30). New York, NY: The Gruyter.

Kang, M., Kim, J. R., \& Cha, H. (2018). From concerned citizens to activists: a case study of 2015 South Korean MERS outbreak and the role of dialogic government communication and citizens' emotions on public activism. Journal of Public Relations Research, 30(5-6), 202-229.

Lecy, J. D., \& Van Slyke, D. M. (2012). Nonprofit sector growth and density: testing theories of government support. Journal of Public Administration Research and Theory, 23(1), 189-214.

Lin, T. H. (2015). Governing natural disasters: state capacity, democracy, and human vulnerability. Social Forces, 93(3), 1267-1300.

Marchesini da Costa, M. (2017). Assessing Government-Nonprofit Collaborations and Nonprofit Density in Brazil. Revista de Administração Pública, 51(3), 330-347.

Marchesini da Costa, M. (2019). Paths for improving non-profit impact on society: lessons from government-non-profit relationships in Brazil. Development in Practice, 29(3), 300-313.

Marcondes, M. M, Sandim, T. L., \& Diniz, A. P. R. (2018, janeiro-março). Transversalidade e Intersetorialidade: mapeamento do debate conceitual no cenário brasileiro. Administração Pública e Gestão Social, 10(1), 1-73.

Mendonça, P. M. E., Alves, M. A., \& Nogueira, F. A. (2013). Arquitetura institucional de apoio às organizações da sociedade civil no Brasil: relatório final eixo Investimento Social Privado corporativo. São Paulo, SP: FGV.https://www.cdes.gov.br/jspui/ bitstream/11451/1019/1/rf_invest_social_priv_corp. pdf

Mendonça, P., Alves, M. A., \& Nogueira, F. (2016). Civil society organisations and the fight for rights in Brazil: analysis of an evolving context and future challenges. Development in Practice, 26(5), 592-605.

Moreira, J. N., Teodósio, A. S. S., \& Ayres, L. C. (2020). Participação das Organizações da Sociedade Civil nas Políticas Públicas Locais: a experiência do Movimento Nossa BH. Perspectivas em Políticas Públicas, 12(24), 109-142.

Najam, A. (2000). The Four C's of Government Third Sector-Government Relations. Nonprofit Management and Leadership, 10(4), 375-396.

O’Donovan, K. (2019). Disaster recovery service delivery: Toward a theory of simultaneous government and voluntary sector failures. Administration \& Society, 51(1), 120-139.

Offe, C. (1984). Dominação de classe e sistema político. Sobre a seletividade das instituições políticas. In C. Offe. (Ed.), Problemas Estruturais Do Estado Capitalista (pp. 140-177). Rio de Janeiro, RJ: Tempo Brasileiro.

Pagotto, L. M., Belinky, A., Macedo, F., \& Yamahaki, C. (2016). Entre o público e o privado: caminhos do alinhamento entre o investimento social privado e o negócio. São Paulo, SP: FGV.

Peci, A., Oquendo, M. I., \& Mendonça, P. (2020). Collaboration, (dis) trust and control in Brazilian manufactured public/non-profit partnerships. Voluntas, 31, 375-389.

Ribeiro, A. C., Andion, C., \& Burigo, F. (2015). Ação coletiva e coprodução para o desenvolvimento rural: um estudo de caso do Colegiado de Desenvolvimento Territorial da Serra Catarinense. Revista de Administração Pública, 49(1), 119-140.

Salamon, L. M. (1987). Of market failure, voluntary failure, and third-party government: Toward a theory of government-nonprofit relations in the modern welfare state. Nonprofit and Voluntary Sector Quarterly, 16(1-2), 29-49.

Salamon, L. M. (1995). Partners in public service: government-nonprofit relations in the modern welfare state. Baltimore, Maryland: John Hopkins Press.

Salamon, L. M., \& Anheier, H. K. (1998). Social origins of civil society: Explaining the nonprofit sector cross-nationally. Voluntas, 9(3), 213-248.

Shaw, R., \& Goda, K. (2004). From disaster to sustainable civil society: the Kobe experience. Disasters, 28(1), 16-40.

Simo, G., \& Bies, A. L. (2007). The role of nonprofits in disaster response: An expanded model of crosssector collaboration. Public Administration Review, 67(s1), 125-142. 
Smillie, I. (1995). The Alms bazaar: altruism under fire; non-profit organizations and international development. London, UK: Intermediation Technology Publications.

Smith, S. R., \& Lipsky, M. (1993). Nonprofit for hire: the Welfare State in the age of contracting. Cambridge, MA: Harvard University Press.

Souza, A. G., \& Oliveira. (2020). De Prestadora de Serviços à Parceira? Como Representantes de OSCs Atuantes na Defesa de Direitos percebem a Lei ${ }^{\circ}$ 13.019/2014. In: A. R. Leichsenring, A. L. Souza, L. Oliveira, L. Vilas Boas, P. Mendonça, A. Viotto \& T. Donnini. (Eds.), Marco Regulatório das Organizações da Sociedade Civil: Visões sobre os primeiros anos de vigência da Lei no 13.019/2014. São Paulo, SP: FGV.

Steinberg, R. (2003). Economic theories of nonprofit organizations. In H. K. Anheier \& A. Ben-Ner, The study of nonprofit enterprise: Theories and approaches. New York, NY: Springer US.

Tilly, C. (2008). Credit and blame. Princeton, Nova Jersey, USA: Princeton University Press.

Titmuss, R. M. (1974). Social policy: An introduction. Crows Nest, Austrália: Allen and Unwin.

Waugh, W. L., Jr., \& Streib, G. (2006). Collaboration and leadership for effective emergency management. Public Administration Review, 66, 131-140.

Waugh, W. L., Jr., \& Sylves, R. T. (2002). Organizing the war on terrorism. Public Administration Review, $62,145-153$.

Weisbrod, B. (1978). The Voluntary Nonprofit Sector. Lanham, Maryland: Lexington Books.

Young, D. R. (2000). Alternative models of government-nonprofit sector relations: Theoretical and international perspectives. Nonprofit and Voluntary Sector Quarterly, 29(1), 149-172.

\section{Mário Aquino Alves}

https://orcid.org/0000-0002-6819-2585

Professor Associado na Escola de Administração de Empresas de São Paulo da Fundação Getulio Vargas (FGV EAESP). E-mail: Mario.Alves@fgv.br

\section{Marcelo Marchesini da Costa}

https://orcid.org/0000-0002-7039-6923

Professor Assistente no Insper Instituto de Ensino e Pesquisa (Insper). E-mail: marcelomc5@insper.edu.br 